\title{
INVARIANCE OF SOLUTIONS TO INVARIANT PARAMETRIC VARIATIONAL PROBLEMS \\ BY
}

JOHN E. BROTHERS ${ }^{1}$

\begin{abstract}
Let $G$ be a compact Lie group of diffeomorphisms of a connected orientable manifold $M$ of dimension $n+1$. Assume the orbits of highest dimension to be connected. Let $\Psi$ be a convex positive even parametric integrand of degree $n$ on $M$ which is invariant under the action of $G$. Let $T$ be a homologically $\Psi$-minimizing rectifiable current of dimension $n$ on $M$, and assume there exists a $G$-invariant rectifiable current $T^{\prime}$ which is homologous to $T$. It is shown that $T$ is $G$-invariant provided $\Psi$ satisfies a symmetry condition which makes it no less efficient for the tangent planes of $T$ to lie along the orbits. This condition is satisfied by the area integrand in case $G$ is a group of isometries of a Riemannian metric on $M$. Consequently, one obtains the corollary that if a rectifiable current $T$ is a solution to the $n$-dimensional Plateau problem in $M$ with $G$-invariant boundary $\partial T$, and if $\partial T$ bounds a $G$-invariant rectifiable current $T^{\prime}$ such that $T-T^{\prime}$ is a boundary, then $T$ is $G$-invariant. An application to the Plateau problem in $\mathbf{S}^{3}$ is given.
\end{abstract}

1. Introduction. Let $G$ be a compact (but not necessarily connected) Lie group of diffeomorphisms of a connected orientable manifold $M$ of dimension $n+1$. Assume the orbits of highest dimension to be connected. Let $\Psi$ be a convex positive even parametric integrand of degree $n$ on $M$ which is invariant under the action of $G$. Let $T$ be a homologically $\Psi$-minimizing rectifiable current of dimension $n$ on $M$, and assume there exists a $G$-invariant rectifiable current $T^{\prime}$ which is homologous to $T$. We show that $T$ is $G$-invariant provided $\Psi$ satisfies a symmetry condition which makes it no less efficient for the tangent planes of $T$ to lie along the orbits. This condition is satisfied by the area integrand in case $G$ is a group of isometries of a Riemannian metric on $M$. Consequently, one obtains the corollary that if a rectifiable current $T$ is a solution to the $n$-dimensional Plateau problem in $M$ with $G$-invariant boundary $\partial T$, and if $\partial T$ bounds a $G$-invariant rectifiable current $T^{\prime}$ such that $T-T^{\prime}$ is a boundary, then $T$ is $G$-invariant.

The first result in this direction was obtained by Fleming and Lawson [L2] for the area integrand in $\mathbf{R}^{n+1}$. They showed that if $G$ is a group of orientation preserving isometries which leave invariant a compact connected oriented $(n-1)$ dimensional submanifold $B$ of $\mathbf{S}^{n} \subset \mathbf{R}^{n+1}$, then there exists an absolutely area

Received by the editors October 31, 1978.

AMS (MOS) subject classifications (1970). Primary 49F22, 49F20; Secondary 53A10, 58A25, 49F10, $28 \mathrm{A75}$.

Key words and phrases. Parametric integrand, $G$-invariant current, homologically $\Psi$-minimizing rectifiable current, Plateau problem.

${ }^{1}$ This work was partially supported by a research grant from the National Science Foundation.

(C) 1980 American Mathematical Society $0002-9947 / 80 / 0000-0504 / \$ 06.25$ 
minimizing $G$-invariant rectifiable current $T$ in $\mathbf{R}^{n+1}$ with $\partial T=B$. Our theorem implies this result for the case where the orbits of highest dimension are connected.

The next result was obtained by Bindschadler [BD] for the area integrand on a Riemannian manifold $M$ under the assumption that $G$ is a group of isometries of $M$ such that the distribution of orthogonal complements to the tangent spaces of the orbits is involutive. He showed that if $T$ is an absolutely area minimizing rectifiable current of dimension $m+1$ on $M$ ( $m$ is the maximal dimension of the orbits) such that there exists a $G$-invariant rectifiable current $T^{\prime}$ with $\partial T^{\prime}=\partial T$, then $T$ is $G$-invariant.

Bindschadler's assumption concerning the orthogonal complements to the tangent spaces of the orbits is, unfortunately, rather restrictive. Although it is satisfied by the actions considered in [L2] (in case $m=n-1$ ), it does not hold for the action of the group $\mathbf{S}^{1}$ of complex numbers of modulus one on the unit sphere $\mathbf{S}^{3} \subset \mathbf{R}^{4}=\mathbf{C}^{2}$ defined by complex scalar multiplication. Our investigation was motivated by a desire to understand this situation, as well as to extend the result of Fleming and Lawson to arbitrary manifolds in the stronger form suggested by Bindschadler in which all solutions to the Plateau problem are invariant.

Our main result is in $\S 4$. The proof is based on a theorem of Federer [F3] which asserts that in codimension one a $\Psi$-minimizing rectifiable current is also a solution to a less restrictive variational problem which allows as candidates for the minimum currents with real coefficients as well as integer coefficients. This allows us to use averaging techniques, which when applied to rectifiable currents that are candidates for the minimum of $\Psi$ yield the more general flat currents, which are thereby also candidates for the minimum. We also make essential use of results in [BD]; in particular, Lemma 3.6 was suggested by [BD, 4.8].

$\$ 5$ contains regularity results for invariant $\Psi$-minimizing currents which are implied by the general regularity theory in [F1] and [SSA].

In $\$ 6$ we apply the lifting theory developed in [BJ1] and [BD] to develop the relationship between invariant $\Psi$-minimizing currents in $M$ and solutions to a related variational problem in $M / G$. This is especially useful if $m=n-1$, in which case the problem of finding invariant minimizing currents in $M$ reduces to that of finding solutions to a one-dimensional variational problem, a procedure previously used in [L2].

$\$ 7$ contains applications of our theory to the discussion of several examples, including the action of $\mathbf{S}^{1}$ on $\mathbf{S}^{3}$ mentioned above. We also discuss the relevance to our results of the counterexample to the question of existence of invariant solutions to the Plateau problem which was given by Federer in [F1].

2. Preliminaries. The purpose of this section is to fix basic notation and terminology, and discuss general concepts which will be used in the paper. Notations which are not explained below may be found in [F1, pp. 669-671]. Basic facts concerning transformation groups may be found in [BG].

2.1. Throughout the paper $M$ and $G$ will be as in $\S 1$. $K$ and $L$ will denote local Lipschitz neighborhood retracts in $M$ which are $G$-invariant, with $K$ compact and $L$ closed. We also assume that $K \cap L$ is a Lipschitz neighborhood retract, and that 
$K$ is the union of a countable family of submanifolds of class 1 of $M$. $k$ will be an integer with $0 \leqslant k \leqslant n+1$.

We denote the tangent space to $M$ at $x \in M$ by $\mathbf{T}_{x}(M)$ and the tangent bundle by $\mathbf{T}(M) . \mathscr{X}(M)$ is the vector space of smooth vector fields on $M ; \mathscr{X}_{G}(M)$ is the subspace of vector fields tangent to the orbits of $G$. If $V$ is a vector space, $\bigwedge_{k}(V)$ denotes the vector space of $k$-vectors in $V . \wedge_{k} \mathbf{T}(M)$ is the $k$-vector bundle of $M$.

2.2. Orbits. For each $x \in M$ the isotropy subgroup at $x$ is

$$
G_{x}=G \cap\{g: g(x)=x\} .
$$

It is well known (see, for example, [BG, IV, 3.1 and VI, 2.4]) that there exists $x_{0} \in M$ such that for each $x \in M, H=G_{x_{0}}$ is conjugate to a subgroup of $G_{x}$. Recalling that if $x$ and $y$ are on the same orbit then $G_{x}$ and $G_{y}$ are conjugate, we call an orbit $G(x)$ principal if $G_{x}$ is conjugate to $H$; denote $m=\operatorname{dim} G\left(x_{0}\right)$. The union $M_{0}$ of the set of principal orbits is open and dense in $M$. Orientability of $M$ implies that the principal orbits are orientable [BG, IV, 3.11]; orient $G / H$. Consider $x \in M$ and a subgroup $H_{x}$ of $G_{x}$ conjugate to $H$. The orbit $G(x)$ is singular if $\operatorname{dim} G_{x} / H_{x}>0$ and exceptional if $\operatorname{dim} G_{x} / H_{x}=0 . G(x)$ is a special exceptional orbit if $G_{x} / H_{x}$ has order 2 . An orbit type is an equivalence class of orbits under the equivalence relation of conjugacy of isotropy subgroups.

It is well known (see, for example, [BG, II, 5.8]) that $M_{0}^{*}=M_{0} / G$ is a smooth manifold of dimension $n+1-m$ and the projection $\pi: M_{0} \rightarrow M_{0}^{*}$ is the projection map of a smooth fibre bundle $\mathfrak{T}_{0}$ with fibre $G / H$ and structure group $\Re(H) / H$ acting by right translation on $G / H$. $(\Re(H)$ is the normalizer of $H$ in $G$.) Furthermore, bundle coordinate maps are equivariant.

It will be useful to provide $M$ with a $G$-invariant Riemannian metric $b$. Such a metric may be obtained from an arbitrary metric $b_{0}$ by averaging $b_{0}$ with respect to the action of $G$. Corresponding to $b$ there is a unique Riemannian metric $b_{\pi}$ on $M_{0}^{*}$ such that for each $x \in M_{0}, \pi_{\sharp} \mid \mathbf{T}_{x}(G(x))^{\perp}$ is an isometry.

We define the volume function $V$ on $M / G$ by

$$
V(z)=\iota(z) \mathcal{H}^{m}(z) \text { for } z \in M / G,
$$

where $\iota(z)$ is the index of a suitable conjugate of $H$ in $G_{x}$ for $x \in z$ in case $z$ is an exceptional orbit, and $\iota(z)=1$ otherwise. $V$ is continuous on $M / G$ and smooth on $M_{0}^{*}$.

2.3. Currents. $\mathbf{M}_{k}(M)$ is the vector space of $k$-dimensional currents $S \in \mathcal{E}_{k}(M)$ with compact support and finite mass $\mathbf{M}(S) . \mathbf{M}_{k}^{\text {loc }}(M)$ is the space of currents $T \in \mathscr{D}_{k}(M)$ such that $\mathbf{M}(T L \gamma)<\infty$ whenever $\gamma \in \mathscr{D}^{0}(M) .\|T\|$ is the variation measure of $T$ and one has the representation

$$
T(\phi)=\int\langle\vec{T}, \phi\rangle d\|T\|
$$

whenever $\phi$ is a continuous differential $k$-form with compact support in $M$, where $\vec{T}(x)$ is a $k$-vector of unit mass for $\|T\|$ almost all $x \in M . F_{k}(M)$ is the vector space of $k$-dimensional flat currents (with compact support) in $M . \Re_{k}(M)$ is the group of $k$-dimensional rectifiable currents in $M$.

$$
\mathscr{F}_{k}(M)=\mathscr{R}_{k}(M)+\partial \mathscr{R}_{k+1}(M)
$$


is the group of $k$-dimensional integral flat currents in $M$.

$$
\mathbf{I}_{k}(M)=\mathscr{R}_{k}(M) \cap\left\{T: \partial T \in \mathscr{R}_{k-1}(M) \text { or } k=0\right\} .
$$

is the group of $k$-dimensional integral currents in $M$. One has the inclusions $\mathbf{I}_{k}(M) \subset \mathscr{R}_{k}(M) \subset \mathscr{F}_{k}(M) \subset \mathbf{F}_{k}(M)$.

$\mathbf{F}_{k}^{\text {loc }}(M)$ and $\mathbf{R}_{k}^{\text {loc }}(M)$ are respectively the sets of locally flat and locally rectifiable currents in $M$.

Whenever $B \subset A \subset M$ we define the vector spaces of $k$-dimensional real flat cycles

$$
\mathbf{Z}_{k}(A, B)=\left\{T: T \in \mathbf{F}_{k}(M), \text { spt } T \subset A, \text { spt } \partial T \subset B \text { or } k=0\right\}
$$

and the subspace of $k$-dimensional real flat boundaries

$$
\mathbf{B}_{k}(A, B)=\left\{T+\partial S: T \in \mathbf{F}_{k}(M) \text {, spt } T \subset B, S \in \mathbf{F}_{k+1}(M) \text {, spt } S \subset A\right\} \text {. }
$$

We define the group of $k$-dimensional integral flat cycles

$$
\mathscr{Z}_{k}(A, B)=\mathbf{Z}_{k}(A, B) \cap \Re_{k}(M)
$$

and the subgroup of $k$-dimensional integral flat boundaries

$$
\mathscr{B}_{k}(A, B)=\left\{T+\partial S: T \in \mathscr{F}_{k}(M) \text {, spt } T \subset B, S \in \mathscr{F}_{k+1}(M) \text {, spt } S \subset A\right\} .
$$

We also define the $k$-dimensional real homology group

$$
\mathbf{H}_{k}(A, B ; \mathbf{R})=\mathbf{Z}_{k}(A, B) / \mathbf{B}_{k}(A, B)
$$

and the $k$-dimensional integral homology group

$$
\mathbf{H}_{k}(A, B ; \mathbf{Z})=\mathscr{Z}_{k}(A, B) / \Re_{k}(A, B) ;
$$

in case $A$ and $B$ are local Lipschitz neighborhood retracts these groups are respectively isomorphic to the singular homology groups of the pair $(A, B)$ with real and integer coefficients [F1, \$4.4].

Let $W$ be a $G$-invariant open subset of $M_{0}$. We will call $T \in \mathscr{D}_{k}(W)$ a local product if for each $x \in W$ there exist an open subset $U$ of $\pi(W)$ containing $\pi(x)$, a bundle coordinate map $\phi_{U}: U \times G / H \rightarrow W$, and $T_{U} \in \mathscr{D}_{k-m}(U)$ such that

$$
T \mid \mathscr{D}_{k}\left(\pi^{-1} U\right)=\phi_{U \sharp}\left(T_{U} \times G / H\right) .
$$

2.4. Integrands. A parametric integrand of degree $k$ on $M$ is a continuous function

$$
\Psi: \wedge_{k} \mathbf{T}(M) \rightarrow \mathbf{R}
$$

satisfying the condition

$$
\Psi(r \alpha)=r \Psi(\alpha) \text { for } \alpha \in \bigwedge_{k} \mathbf{T}(M), r>0 .
$$

$\Psi$ is positive if

$$
\Psi(\alpha)>0 \text { for } 0 \neq \alpha \in \wedge_{k} \mathbf{T}(M)
$$

$\Psi$ is even if

$$
\Psi(-\alpha)=\Psi(\alpha) \text { for } \alpha \in \bigwedge_{k} T(M)
$$

$\Psi$ is convex if

$$
\Psi(\alpha+\beta) \leqslant \Psi(\alpha)+\Psi(\beta) \text { for } x \in M \text { and } \alpha, \beta \in \wedge_{k} \mathbf{T}_{x}(M)
$$


$\Psi$ is called an integrand of class $q$ if the restriction of $\Psi$ to the set of nonzero elements of $\bigwedge_{k} \mathbf{T}(M)$ is of class $q$. We associate with each $x \in M$ the constant coefficient integrand

$$
\Psi_{x}=\Psi \mid \mathrm{T}_{x}(M) .
$$

Whenever $\Psi$ is positive and $T \in \mathbf{M}_{k}^{\text {loc }}(M)$, the function which corresponds $\Psi(\vec{T}(x))$ with $x \in M$ is $\|T\|$ integrable; one denotes

$$
\int \Psi \circ \vec{T} d\|T\|=\int_{T} \Psi .
$$

The parametric area integrand of degree $k$ on $M$ is the function

$$
\Psi(\alpha)=|\alpha|, \quad \alpha \in \bigwedge_{k} \mathbf{T}(M) .
$$

In this case $\int_{T} \Psi=\mathbf{M}(T)$ whenever $T \in \mathscr{R}_{k}(M)$.

Let $\Psi$ be a parametric integrand of degree $k$ and class 2 on $M$. One says that $\Psi$ satisfies a parametric Legendre condition if for each compact subset $A$ of $M$ there exists $c_{A}>0$ such that for each $x \in A$,

$$
D^{2} \Psi_{x}(\alpha)(\beta, \beta) \geqslant c_{A}|\beta \wedge \alpha|^{2} /|\alpha|^{3}
$$

whenever $\alpha, \beta \in \wedge_{k} \mathbf{T}_{x}(M)$ and $\alpha \neq 0$. (Here $D^{2} \Psi_{x}$ is the second differential of $\Psi_{x}$.) The area integrand satisfies this condition with $c_{A}=1$. If $\Psi$ satisfies this condition then Taylor's Theorem implies that $\Psi$ is convex; in fact, $\Psi$ is elliptic [F1, 5.1.2]. Moreover, the argument in [F1, 5.1.2] can be adapted to show that ellipticity is equivalent to a parametric Legendre condition for $k=n$ or $k=1$.

Let $\Psi$ be a parametric integrand of degree $k$ on $M$ and $A, B, C \subset M$ with $B \subset A$. A current $T$ will be called homologically $\Psi$-minimizing in $C$ with respect to $(A, B)$ if $T \in \Re_{k}(M)$, spt $T \subset C$, and

$$
\int_{T} \Psi \leqslant \int_{T+Q} \Psi
$$

whenever $Q \in \mathscr{B}_{k}(A, B)$ with spt $Q \subset C$.

Suppose $A, B, C$ are local Lipschitz neighborhood retracts, $B$ is relatively closed in $A$ and $C$ is compact. The following existence theorem is implied by the discussion in [F3, 5.9]: Let $\Psi$ be a positive convex integrand. If $T_{0} \in \Re_{k}(M)$ with spt $T_{0} \subset C$, then there exists $T \in \Re_{k}(M)$ such that $T$ is homologically $\Psi$-minimizing in $C$ with respect to $(A, B)$ and

$$
T-T_{0} \in \mathscr{B}_{k}(A, B) .
$$

We remark that if $T$ is absolutely $\Psi$-minimizing with respect to $(C, C \cap B)$ as defined in [F1, 5.1.6], then $T$ is homologically $\Psi$-minimizing in $C$ with respect to $(A, B)$ which in turn implies that $T$ is homologically $\Psi$-minimizing with respect to $(C, C \cap B)$. Neither of the reverse implications holds in general; however, it is true that if $T$ is homologically $\Psi$-minimizing with respect to $(M, B)$ then $T$ is homologically $\Psi$-minimizing in $C$ with respect to $(M, B)$ whenever $C \supset$ spt $T$.

Finally, we observe that if $\Psi$ is an elliptic integrand on $\mathbf{R}^{n+1}, A$ is a convex subset of $\mathbf{R}^{n+1}$ and $B \subset A$, then $T \in \mathcal{R}_{n}(M)$ is absolutely $\Psi$-minimizing with respect to $\left(\mathbf{R}^{n+1}, B\right)$ if and only if $T$ is absolutely $\Psi$-minimizing with respect to 
$(A, B)$. This follows directly from the definition of "elliptic integrand" [F1, 5.1.2] and is useful in application of our results in $\mathbf{R}^{n+1}$.

2.5. Invariant integrands. The action of $G$ on $M$ induces an action on $\mathbf{T}(M)$ and hence an action on $\wedge_{k} \mathbf{T}(M)$. For each $x \in M_{0}$ we choose $0 \neq \alpha \in \wedge_{m} \mathbf{T}_{x}(G(x))$ and define for $k \geqslant m$,

$$
\mathfrak{V}_{k, x}=\left\{\alpha \wedge \beta: \beta \in \wedge_{k-m} \mathbf{T}_{x}(M)\right\}
$$

$\mathfrak{V}_{k, x}$ is clearly independent of the choice of $\alpha$ and is a linear subspace of $\wedge_{k} \mathbf{T}_{x}(M)$ of dimension

$$
\begin{gathered}
\nu=\left(\begin{array}{c}
n+1-m \\
k-m
\end{array}\right) . \\
\mathscr{V}_{k}=\cup\left\{\mathscr{V}_{k, x}: x \in M_{0}\right\}
\end{gathered}
$$

is a $G$-invariant subbundle of $\bigwedge_{k} \mathbf{T}\left(M_{0}\right)$.

Let $\Psi$ be a parametric integrand of degree $k$ on $M$. We will say that $\Psi$ is $G$-invariant provided

(i) $\Psi$ is invariant under the action of $G$ on $\wedge_{k} T(M)$, and

(ii) there exists a $G$-invariant continuous linear bundle map

$$
\Pi_{\Psi}: \bigwedge_{k} \mathbf{T}\left(M_{0}\right) \rightarrow \mathscr{V}_{k}
$$

such that

$$
\Pi_{\Psi} \mid \mathscr{V}_{k}=\mathbf{1}_{\Upsilon_{k}}
$$

and

$$
\Psi \circ \Pi_{\Psi}(\alpha) \leqslant \Psi(\alpha) \quad \text { whenever } \alpha \in \bigwedge_{k} \mathbf{T}\left(M_{0}\right),
$$

with strict inequality for $\alpha \notin \mathcal{V}_{k}$.

We extend $\Pi_{\Psi}$ to $\bigwedge_{k} \mathbf{T}(M)$ so that $\Pi_{\Psi} \mid \bigwedge_{k} \mathbf{T}(M) \sim \wedge_{k} \mathbf{T}\left(M_{0}\right)=0$, and denote $\Pi_{\Psi, x}=\Pi_{\Psi} \mid \wedge_{k} \mathbf{T}_{x}\left(M_{0}\right)$ for $x \in M_{0}$. We also observe that (i) is equivalent to $G$-invariance of the integral of $\Psi$ : If $T \in \mathbf{M}_{k}^{\text {loc }}(M)$ then

$$
\int_{g_{\sharp} T} \Psi=\int_{T} \Psi \quad \text { whenever } g \in G
$$

(Compare [F1, 5.1.1].) Note that the area integrand will satisfy (ii) if $\Pi_{\Psi}$ is the orthogonal projection onto $\mathfrak{V}_{k}$.

In case $\Psi$ is a positive integrand of class 2 which satisfies a parametric Legendre condition and is such that (i) holds, Taylor's Theorem can be used to show that (ii) is equivalent to the requirement that for each $x \in M_{0},\left\{D \Psi_{x}(\alpha): 0 \neq \alpha \in \mathcal{V}_{k, x}\right\}$ spans a linear subspace of $\bigwedge_{k} \mathbf{T}_{x}\left(M_{0}\right)^{*}$ of dimension not greater than $\nu$.

Next we suppose there exists a smooth invariant unit $\boldsymbol{m}$-vector field $\mathrm{v}$ on $\boldsymbol{M}_{\mathbf{0}}$ such that for $x \in M_{0}, \mathrm{v}(x)$ is tangent to $G(x)$. Let $\Psi$ be a parametric integrand of degree $k+m$ and class $q$ on $M_{0}$ such that $g^{\sharp} \Psi=\Psi$ for $g \in G$. We define a parametric integrand $\Psi^{*}$ of degree $k$ and class $q$ on $M_{0}^{*}$ as follows: Let $z \in M_{0}^{*}, x \in z$ and $\lambda_{x}$ : $\mathbf{T}_{z}\left(M_{0}^{*}\right) \rightarrow \mathbf{T}_{x}\left(M_{0}\right)$ be such that $\pi_{\sharp} \circ \lambda_{x}$ is the identity. Then

$$
\Psi^{*}(\alpha)=V(z) \Psi\left(\wedge_{k} \lambda_{x}(\alpha) \wedge v(x)\right) \text { for } \alpha \in \wedge_{k} \mathrm{~T}_{z}\left(M_{0}^{*}\right)
$$


It is clear that $\Psi^{*}(\alpha)$ does not depend on the choice of $\lambda_{x}$ or $x \in z$. Smoothness follows through use of local cross sections. Finally, we remark that in case $\Psi$ is even, existence of $\Psi^{*}$ does not depend on the existence of $\mathbf{v}$.

\section{Invariant flat currents.}

3.1. If $\xi \in \mathscr{X}(M)$ and $A$ is a smooth tensor field on $M$, then $L_{\xi} A$ denotes the Lie derivative of $A$ with respect to $\xi$. For each current $T \in \mathscr{D}_{k}(M)$ we define the Lie derivative $L_{\xi} T \in \mathscr{D}_{k}(M)$ so that

$$
\left(L_{\xi} T\right)(\phi)=-T\left(L_{\xi} \phi\right) \quad \text { for } \phi \in \mathscr{Q}^{k}(M) .
$$

Lemma. If $\xi \in \mathscr{X}(M)$ and $T \in \mathscr{D}_{k}(M)$, then

$$
\partial(T \wedge \xi)=(\partial T) \wedge \xi+(-1)^{k-1} L_{\xi} T
$$

Proof. Using $\iota_{\xi}$ to denote interior multiplication by $\xi[K N$, p. 35] we have $\xi(x)\lrcorner \omega=(-1)^{k} l_{\xi(x)} \omega$ for $\omega \in \wedge^{k+1} \mathbf{T}_{x}(M)$. Consequently, for $\phi \in \mathscr{D}^{k}(M)$,

$$
\left.\left.L_{\xi} \phi=\iota_{\xi}(d \phi)+d \iota_{\xi} \phi=(-1)^{k}[\xi\lrcorner d \phi-d(\xi\lrcorner \phi\right)\right]
$$

hence our identity follows from the definition

$$
T \wedge \xi(\psi)=T(\xi\lrcorner \psi) \quad \text { for } \psi \in \mathscr{Q}^{k+1}(M) .
$$

3.2. Let $\mu$ be the Haar measure on $G$ with $\mu(G)=1$, and denote

$$
\Re_{*}=\mathbf{M}_{*}(M)+\partial \mathbf{M}_{*}(M) \text {. }
$$

There exists a chain map $A: \Re_{*} \rightarrow \Re_{*}$ characterized by the formula

$$
A(T)(\phi)=\int_{G} g_{\sharp} T(\phi) d \mu g \quad \text { for } T \in \mathfrak{T}_{k}, \phi \in \mathcal{E}^{k}(M) ;
$$

compare [BD, 4.4]. We also denote $A(T)=T_{G}$.

Lemma. (i) $T_{G}$ is $G$-invariant whenever $T \in \Re_{*}$.

(ii) $A: \mathbf{F}_{*}(M) \cap \mathfrak{T}_{*} \rightarrow \mathbf{F}_{*}(M) \cap \mathfrak{R}_{*}$.

(iii) Let $\Psi$ be a convex positive integrand of degree $k$ such that $g^{\sharp} \Psi=\Psi$ for $g \in G$. If $T \in \mathbf{M}_{k}(M)$, then

$$
\int_{T_{G}} \Psi<\int_{T} \Psi
$$

Proof. (i) and (ii) follow from the definition of $A$ and the fact that $\partial \circ A=$ $A \circ \partial$. (iii) follows from [F3, 2.1] and the observation that if $\Psi>\phi \in \mathscr{D}^{k}(M)$, then $\Psi \geqslant g^{\sharp} \phi$ for $g \in G$ and thus

$$
T_{G}(\phi)=\int_{G} T\left(g^{\sharp} \phi\right) d \mu g<\int_{T} \Psi .
$$

3.3. Lemma. Suppose $T \in \mathbf{B}_{n}(M, L)$ is $G$-invariant. Then $T \wedge \xi=0$ whenever $\xi \in \mathscr{X}_{G}(M)$ with spt $\xi \subset M_{0} \sim L$.

Proof. Choose $S \in \mathbf{F}_{n+1}(M)$ with $\operatorname{spt}(\partial S-T) \subset L$. Then $S_{G} \in \mathbf{F}_{n+1}(M)$ and $\operatorname{spt}\left(\partial S_{G}-T\right) \subset L$ since $L$ is invariant. Suppose $\xi_{G} \in \mathcal{X}_{G}(M)$ is induced on $M$ by the action of a one-parameter subgroup of $G$. Then $L_{\xi_{G}} S_{G}=0$, hence 3.1 implies 
that $\left(\partial S_{G}\right) \wedge \xi_{G}=0$ and so $\operatorname{spt}\left(T \wedge \xi_{G}\right) \subset L$. From this we conclude that $T \wedge \xi=$ 0 because the set of vector fields $\xi_{G}$ spans $\mathcal{X}_{G}\left(M_{0}\right)$.

3.4. Remark. Simple examples in $\mathbf{R} \times \mathbf{S}^{1}$ show that if $S$ does not exist, then the conclusion of Lemma 3.3 may not be true even if $\partial T=0$.

3.5. Lemma. Suppose $T \in \mathbf{F}_{n}(M)$ is $G$-invariant.

(i) If spt $T \cap M_{0}=\varnothing$, then $T=0$.

(ii) If $\mathbf{M}(T)<\infty$, then $\|T\|\left(M \sim M_{0}\right)=0$.

Proof. (ii) follows from (i) because $M \sim M_{0}$ is closed and $T L\left(M \sim M_{0}\right) \in$ $\mathrm{F}_{n}(M)$ by [F1, 4.1.17].

Assuming spt $T \cap M_{0}=\varnothing$ we use [BG, VI, 2.4, 2.5 and IV, 1.2] to infer that spt $T \subset M_{1} \cup \cdots \cup M_{\alpha}$, where each $M_{i}$ is a smooth proper submanifold of $M$ and is the union of the set of all orbits corresponding to a fixed orbit type. Now lower semicontinuity of orbit type [BG, II, 5.5] implies that if $M_{i}$ is the union of the set of all orbits of a fixed special exceptional orbit type, then $M_{i}$ is a relatively open subset of $M \sim M_{0}$. We will show that spt $T \cap M_{i}=\varnothing$, hence infer using [BG, IV, 3.8 and 3.10] that $\mathcal{H}^{n}($ spt $T)=0$, which implies by [F1, 4.1.20 and 2.10.6] that $T=0$.

In order to show that spt $T \cap M_{i}=\varnothing$ it will suffice to show that $T L f=0$ whenever $f \in \mathscr{D}^{0}(M)$ is $G$-invariant with spt $f \subset M_{i} \cup M_{0}$. We can also assume $\pi\left(\operatorname{spt} f \mid M_{i}\right)$ to lie in an orientable open subset $U$ of the manifold $\pi\left(M_{i}\right)$. Fixing $f$ we infer from the second proposition on [F1, p. 373] that $T L f$ corresponds to $T_{f} \in$ $\mathrm{F}_{n}\left(M_{i}\right)$. Consequently, $T\left\llcorner f=0\right.$ unless $\operatorname{dim} M_{i}=n$; assuming this to be the case we observe that for $\mathcal{H}^{n-m}$ almost all $z \in U$ the slice $T_{f}(z)=\left\langle T_{f}, \pi \mid M_{i}, z\right\rangle \in \mathbf{F}_{m}(z)$ [F1, 4.3.1]. Moreover, $T_{f}(z)$ is $G$-invariant by [F1, 4.3.2] and we infer using Lemma 3.1 that $\partial T_{f}(z)=0$, hence $T_{f}(z)$ represents a real $m$-dimensional homology class of $z$. But $z$ is not orientable [BG, IV, 3.11], hence $T_{f}(z)=0$ and we conclude using [F1, 4.3.2] that $T_{f}=0$.

3.6. Lemma. Suppose $T \in \mathbf{F}_{n}(M) \cap \mathbf{M}_{n}(M)$ and $T^{\prime} \in \mathbf{F}_{n}(M)$ are such that

(i) $T-T^{\prime} \in \mathbf{B}_{n}(M, L)$,

(ii) $g_{\sharp} T^{\prime}=T^{\prime}$ whenever $g \in G$,

(iii) $T^{\prime} \wedge \xi=0$ whenever $\xi \in \mathscr{X}_{G}(M)$ with spt $\xi \subset M_{0} \sim L$, and

(iv) there exists $\xi_{0} \in X_{G}(M)$ with spt $\xi_{0} \subset M_{0} \sim L$ such that $T \wedge \xi_{0} \neq 0$.

Then $T-T_{G} \in \mathbf{B}_{n}(M, L)$ and

$$
\int_{T_{G}} \Psi \leqslant \int_{T} \Psi
$$

whenever $\Psi$ is a convex positive $G$-invariant integrand of degree $n$ on $M$.

Proof. We choose a $G$-invariant open set $U$ with compact closure in $M_{0} \sim L$ such that spt $\xi_{0} \subset U$; denote $T_{0}=T\llcorner U$. Referring to 2.5 we see that

$$
\sup \left\{\left\|\Pi_{\Psi, x}\right\|: x \in U\right\}<\infty,
$$

hence

$$
\Pi T_{0}=\left\|T_{0}\right\| \wedge \Pi_{\Psi} \vec{T}_{0} \in \mathbf{M}_{n}(M)
$$


and we infer using Lemma 3.2 that

$$
\int_{\left(\Pi T_{0}\right)_{G}} \Psi \leqslant \int_{\Pi T_{0}} \Psi<\int_{T_{0}} \Psi
$$

Now the $G$-invariance and linearity oi $\Pi_{\Psi}$ imply that $\left(\Pi T_{0}\right)_{G}=\Pi\left(T_{0 G}\right)$. Thus our assertion will follow if we can show that $\Pi\left(T_{0 G}\right)=T_{0 G}$ because $T_{G}=T_{0 G}+$ $\left(T-T_{0}\right)_{G}$, hence

$$
\int_{T_{G}} \Psi<\int_{T_{0}} \Psi+\int_{T-T_{0}} \Psi=\int_{T} \Psi
$$

Choose $S \in \mathbf{F}_{n+1}(M)$ with $\operatorname{spt}\left[\partial S-\left(T-T^{\prime}\right)\right] \subset L$ and observe that

$$
\operatorname{spt}\left[\partial S_{G}-\left(T_{G}-T^{\prime}\right)\right] \subset L
$$

since $L$ is invariant, hence $\operatorname{spt}\left[\partial\left(S-S_{G}\right)-\left(T-T_{G}\right)\right] \subset L$ and thus $T-T_{G} \in$ $\mathbf{B}_{n}(M, L)$. Further, in view of Lemma 3.2 we can apply Lemma 3.3 to obtain

$$
0=\left(T_{G}-T^{\prime}\right) \wedge \xi=T_{G} \wedge \xi
$$

whenever $\xi \in \mathscr{X}_{G}(M)$, spt $\xi \subset M_{0} \sim L$, which implies that on $M_{0} \sim L, \Pi_{\Psi} \vec{T}_{G}=$ $\vec{T}_{G}$. Finally, since $\vec{T}_{G}\left|U=\left(T_{0 G}\right) \rightarrow\right| U$, we conclude that

$$
\Pi\left(T_{0 G}\right)=T_{0 G} \text {. }
$$

\section{4. $\Psi$-minimizing currents.}

4.1. Lemma. Let $U$ and $Y$ be oriented Riemannian manifolds with $Y$ connected and $\operatorname{dim} Y=m$. Suppose $T \in \mathbf{F}_{k+m}^{\mathrm{loc}}(U \times Y) \cap \mathbf{M}_{k+m}^{\text {loc }}(U \times Y)$ is such that

(i) $\vec{T} \wedge \xi=0$ whenever $\xi \in \mathcal{X}(U \times Y)$ is such that $\xi \mid\{z\} \times Y$ is tangent to $\{z\} \times Y$ for $z \in U$, and

(ii) $\partial T=0$.

Then there exists $R \in F_{k}^{\text {loc }}(U) \cap M_{k}^{\text {loc }}(U)$ such that $T=R \times Y$. Furthermore, if $T \in \mathcal{R}_{k+m}^{\text {loc }}(U \times Y)$, then $R \in \mathcal{R}_{k}^{\text {loc }}(U)$.

Proof. The existence of $R$ is obtained in [BD, 3.5]. If $p$ and $q$ denote the projections of $U \times Y$ on $U$ and $Y$, respectively, we infer from the definition of the slice $\langle R \times Y, q, y\rangle$ corresponding to $y \in Y[F 1,4.3 .1]$ that $R=p_{\sharp}\langle R \times Y, q, y\rangle$. Thus by [F1, 4.3.6] $R$ is rectifiable if $T$ is rectifiable.

4.2. Corollary. Suppose there exists $T \in \mathbf{F}_{k+m}\left(M_{0}\right) \cap \mathbf{M}_{k+m}\left(M_{0}\right)$ which is $G$-invariant and is such that $\|T\|\left(M_{0} \sim \operatorname{spt} \partial T\right) \neq 0$ and $T \wedge \xi=0$ whenever $\xi \in$ $\mathcal{X}_{G}(M)$ with spt $\xi \subset M_{0}$. Then the action of $G$ preserves the orientation of $G / H$.

Proof. Let $U$ be an open subset of the open set $M_{0}^{*} \sim \pi(\operatorname{spt} \partial T)$ such that $T L U \neq 0$ and there exists a coordinate map $\phi_{U}: U \times G / H \rightarrow \pi^{-1} U$. Applying Lemma 4.1 we infer that

$$
T \mid \mathscr{D}^{k+m}\left(\pi^{-1} U\right)=\phi_{U \sharp}(R \times G / H) .
$$

Our assertion now follows from the invariance of $T$ and the equivariance of $\phi_{U}$. 
4.3. Lemma. Let $\Psi$ be a convex positive $G$-invariant integrand of degree $n$ on $M$. Assume $0 \neq T \in \mathbf{F}_{n}(M) \cap \mathbf{M}_{n}(M)$ is such that spt $T \subset K$ and the following hold:

(i) $\int_{T} \Psi=\inf \left\{\int_{T+Q} \Psi: Q \in \mathbf{B}_{n}(M, L)\right.$, spt $\left.Q \subset K\right\}$.

(ii) $\|T\|(B)=0$ where $B=M_{0} \cap$ spt $\partial T \sim L$.

(iii) There exists $T^{\prime} \in \mathbf{F}_{n}(M) \cap \mathbf{M}_{n}(M)$ with $T-T^{\prime} \in \mathbf{B}_{n}(M, L)$ such that $T^{\prime}$ is $G$-invariant, $\left\|T^{\prime}\right\|(M \sim B) \neq 0$, and $T^{\prime} \wedge \xi=0$ whenever $\xi \in \mathscr{X}_{G}(M)$ with spt $\xi \subset$ $M_{0} \sim L$.

Then the following are true:

(1) $T \mid \mathscr{D}^{n}\left(M_{0} \sim(B \cup L)\right)$ is a local product.

(2) $\|T\|\left[M \sim\left(M_{0} \sim(B \cup L)\right)\right]=0$.

(3) $T$ is $G$-invariant.

Proof. Note that $B$ is $G$-invariant because $\operatorname{spt}\left(\partial T-\partial T^{\prime}\right) \subset L$ and spt $\partial T^{\prime}$ is invariant. Now $T^{\prime} \mathrm{L}(M \sim L) \neq 0$ because if spt $T^{\prime} \subset L$ then $T=0$. Consequently, since $\left\|T^{\prime}\right\|\left(M \sim M_{0}\right)=0$ by Lemma 3.5 , and $M_{0} \cap$ spt $\partial T^{\prime} \subset B \cup L$, Corollary 4.2 implies that the action of $G$ preserves the orientation of $G / H$.

Next we infer using Lemma 3.6 and (i) that

$$
\int_{T_{G}} \Psi=\int_{T} \Psi
$$

because $K$ is invariant, and therefore $T \wedge \xi=0$ whenever $\xi \in \mathscr{X}_{G}(M)$ with spt $\xi \subset M_{0} \sim L$. Let $U$ be an open subset of the open set $M_{0}^{*} \sim \pi(B \cup L)$ such that there exists an equivariant coordinate map $\phi_{U}: U \times G / H \rightarrow \pi^{-1} U$; assume also that $U$ has compact closure in $M_{0}^{*} \sim \pi(B \cup L)$. It follows from Lemma 4.1 that $T \mid \mathscr{D}^{n}\left(\pi^{-1} U\right)$ is a product, hence (1) is clear. Moreover, it is also clear that $T L \pi^{-1} U$ is invariant; choosing a locally finite open cover of $M_{0}^{*} \sim \pi(B \cup L)$ consisting of open sets such as $U$ one uses a subordinate partition of unity to show that $T \mathrm{~L}\left(M_{0} \sim(B \cup L)\right)$ is invariant. Thus (3) will follow once (2) is verified.

For the proof of (2) we first observe that $T L L \in \mathbf{B}_{n}(M, L)$ by [F1, 4.1.17], hence because $\Psi$ is positive and $\mathcal{F}^{n}(B)=0$, we have

$$
\left\|T_{G}\right\|(B \cup L) \leqslant\|T\|(B \cup L)=0 .
$$

Thus since $T L\left(M_{0} \sim(B \cup L)\right)=T_{G} \mathrm{~L}\left(M_{0} \sim(B \cup L)\right)$, we have

$$
\int_{T\left\llcorner\left(M \sim M_{0}\right)\right.} \Psi=\int_{T_{G}\left\llcorner\left(M \sim M_{0}\right)\right.} \Psi=0
$$

by Lemma 3.5 and (*). Finally, positivity of $\Psi$ implies that $T L\left(M \sim M_{0}\right)=0$.

4.4. TheOREM. Let $\Psi$ be a convex positive even $G$-invariant integrand of degree $n$ on $M$ and $T \in \Re_{n}(M)$ be homologically $\Psi$ minimizing in $K$ with respect to $(M, L)$. Assume that conditions (ii) and (iii) of Lemma 4.3 are satisfied. Then the conclusions of Lemma 4.3 hold for $T$.

Proof. With the help of [F1, 4.1.12, 4.1.15 and 4.1.29] we observe that

$$
\mathbf{B}_{n, K}(M, L)=\mathbf{F}_{n, K}(M, L) \cap \mathbf{B}_{n}(M, L)=\mathbf{B}_{n}(M, L) \cap\{Q: \text { spt } Q \subset K\}
$$

and

$$
\mathscr{P}_{n, K}(M, L)=\mathscr{F}_{n, K}(M, L) \cap \mathscr{B}_{n}(M, L)=\mathscr{B}_{n}(M, L) \cap\{Q: \operatorname{spt} Q \subset K\} .
$$


Using this we apply [F3, 2.13 and 5.10] to infer that

$$
\int_{T} \Psi=\inf \left\{\int_{T+Q} \Psi: Q \in \mathbf{B}_{n}(M, L), \text { spt } Q \subset K\right\}
$$

consequently, Lemma 4.3 applies.

4.5. RemARKs. (1) Suppose $M=\mathbf{R}^{n+1}$ and $G$ is a group of linear transformations. In case $\partial T$ is $G$-invariant and rectifiable we can take $T^{\prime}$ to be the cone over $\partial T$, $T^{\prime}=\delta_{0} * \partial T$ [F1, p. 365], which is clearly invariant; moreover, $T-T^{\prime} \in$ $\mathbf{B}_{n}\left(\mathbf{R}^{n+1}\right)$ because $\partial T^{\prime}=\partial T$. If $\xi \in \mathscr{X}_{G}(M)$ we infer with the help of [FF, 8.16] that $\partial T \wedge \xi=0$, from which it follows that $T^{\prime} \wedge \xi=0$.

On the other hand, invariance of $\partial T$ does not in general imply existence of $T^{\prime}$ even if $\mathbf{H}_{n-1}(M ; \mathbf{R})=0$. In 7.2 we will show that if $G=\mathbf{S}^{1}$ acts on $M=\mathbf{S}^{3} \subset \mathbf{C}^{2}$ by means of complex scalar multiplication and $\partial T=c_{1}+c_{2}$ where

$$
c_{1}=\mathbf{S}^{3} \cap \mathbf{C} \times\{0\}, \quad c_{2}=\mathbf{S}^{3} \cap\{0\} \times \mathbf{C}
$$

with their usual orientations when regarded as subsets of $\mathbf{C}$, then $T$ cannot be $\mathbf{S}^{1}$-invariant. Furthermore, since this action is the restriction to $\mathbf{S}^{\mathbf{3}}$ of an action of $\mathbf{S}^{1}$ on $\mathbf{R}^{4}$, this example shows that our theorem does not hold in codimension 2 for the case where $M=\mathbf{R}^{4}, K=\mathbf{S}^{3}$ and $L=\varnothing$.

Finally, we observe that if $G$ is a group of linear transformations of $M=\mathbf{R}^{n+1}$, $T \in \mathbf{F}_{n}(M)$ is $G$-invariant, and spt $\partial T \cap M_{0}$ is compact, then spt $\partial T \subset M_{0}$. (Compare [L2, Proposition 4.4].) Indeed, $\partial T=S_{0}+S_{1}$ with $S_{0}, S_{1} \in F_{n-1}(M)$, spt $S_{0}$ $\subset M_{0}$, spt $S_{1} \subset M \sim M_{0} . S_{1}$ is invariant hence so is $S_{1}^{\prime}=\delta_{0} \nVdash S_{1} \in F_{n}(M)$; moreover, spt $S_{1}^{\prime} \subset M \sim M_{0}$ and therefore Lemma 3.5 implies that $\mathbf{S}_{1}^{\prime}=0$. This is not, however, true in general. Simple examples can be found in $M=\mathbf{S}^{3}$ with $G=\mathbf{S}^{1}$ as in 7.2.

(2) If $M$ is a manifold with boundary $N$ with $G$ acting smoothly on $M \cup N$, then $M \cup N \subset M^{\prime}$ where $M^{\prime}$ is an orientable $(n+1)$-dimensional manifold to which the action of $G$ can be extended and is such that $M \cup N$ is a smooth deformation retract of $M^{\prime}$. Thus

$$
\mathbf{B}_{n}(M \cup N)=\mathbf{B}_{n}\left(M^{\prime}\right) \cap\{Q: \text { spt } Q \subset M \cup N\}
$$

and one can apply the theorem with $M$ replaced by $M^{\prime}$ and $K, L \subset M \cup N$ to obtain the analogous result for manifolds with boundary.

(3) With $M, N, M^{\prime}$ as in (2) we discuss the case where the dimension of the principal orbits (in $M$ ) is $n$. It is easy to see that the action of $G$ preserves the orientation of $M$ if and only if the action preserves orientation of the principal orbits; assume this to be the case. By [BG, IV, 3.1 and 3.11], $(M \cup N)_{0}^{*}$ is connected, hence each two principal orbits are homotopic and, when suitably oriented, represent the same homology class $\mathbf{P} \in \mathbf{H}_{n}(M \cup N ; \mathbf{Z})$. In case there exists a nonprincipal orbit $z \in M \cup N / G$ it follows from the existence of linear tubes [BG, VI, 2.4] that there is an $n$-dimensional rectifiable cycle $S_{z}$ supported on $z$ which is homotopic to an oriented principal orbit $z^{\prime} \in \mathbf{P}$. But unless $z$ is a special exceptional orbit $\operatorname{dim} z<n$ [BG, IV, 3.8 and 3.10], hence $S_{z}=0=\mathbf{P}$. If $z$ is special exceptional then $z$ is not orientable [BG, IV, 3.11], hence again $S_{z}=0=\mathbf{P}$. 
Conversely, if all orbits in $M^{\prime}$ are principal and $T \in \mathbf{B}_{n}(M \cup N)$ with spt $T \in M$ $\cup N / G$, then one can use the lifting theory in [BD, §3] (see also 6.1) to show that spt $T \in B_{0}\left(M^{\prime} / G\right)$, which is impossible. We therefore conclude that $\mathbf{P} \neq 0$ if and only if all orbits are principal. (See [BG, IV, §8] also.)

Suppose $M \cup N$ is compact with $\mathbf{P} \neq 0$. It follows from Theorem 4.4 and the remarks in (2) that $T \in \mathbf{P}$ has least $n$-dimensional area in $\mathbf{P}$ if and only if spt $T$ is an orbit with

$$
V(\operatorname{spt} T)=\inf V \mid(M \cup N) / G .
$$

For example, applying this to the flat $(n+1)$-dimensional torus $\mathbf{T}^{n+1}$ with $\mathbf{P}$ the homology class containing $\mathbf{T}^{n} \times\left\{x_{0}\right\}, x_{0} \in \mathbf{S}^{\mathbf{1}}$, we see that $T \in \mathbf{P}$ has least area if and only if

$$
T=\mathbf{T}^{n} \times\{x\}, \quad x \in \mathbf{S}^{1} .
$$

Finally, turning to the case where $M$ is aribitrary and the action of $G$ preserves orientation of the principal orbits, we assume $V$ to have a local minimum at $z_{0} \in M_{0}^{*}$. Choose a closed arc $I^{*} \subset M_{0}^{*}$ such that $V \mid I^{*}$ has a minimum at $z_{0} \in$ int $I^{*}$, and denote $I=\pi^{-1} I^{*}$. Let $T_{0}$ be a current obtained by orienting $z_{0}$. Then $T_{0} \in \mathbf{P} \in \mathbf{H}_{n}(I ; \mathbf{Z})$, and applying the preceding discussion with $M \cup N=I$ we infer that $T_{0}$ is of least area in P. It follows from [F1, p. 525] that $z_{0}$ is minimal and stable. (It is of interest here to recall that if $z$ is a special exceptional orbit then $z$ is minimal [HL, §3]; however, we do not know whether $z$ is in general stable.)

Simple examples of the above can be found in surfaces of revolution in $\mathbf{R}^{3}$ obtained by rotating the graph $\{(x, f(x)): x \in(a, b)\}$ of a smooth positive function $f:(a, b) \rightarrow \mathbf{R}$ about the $x$-axis. The meridian circle corresponding to $x \in(a, b)$ is a geodesic if and only if $f^{\prime}(x)=0$, and is a stable geodesic if and only if $f$ has a local minimum at $x$. In this case, $V(z)=2 \pi f(z)$ for $z \in M / \mathbf{S}^{1}=(a, b)$.

On the other hand, consider the action of $S^{1}$ on $S^{3}$ mentioned in (1). The orbits of this action are all principal, are all geodesics, and are all unstable. However, $V$ is constant, $V=2 \pi$. This, together with the example discussed in (1), illustrates the difficulties which are evidently inherent in the problem of extending our results to codimensions greater than one.

\section{Regularity.}

5.1. Let $\Psi$ be a positive integrand of degree $m+1$ and class 3 on $M_{0}$ such that

(i) $\Psi$ is invariant under the action of $G$ on $\bigwedge_{m+1} \mathbf{T}(M)$, and

(ii) for each compact subset $A$ of $M_{0}$ there exists $c_{A}>0$ such that for each $x \in A$,

$$
D^{2} \Psi_{x}(\alpha)(\beta, \beta) \geqslant c_{A}|\beta \wedge \alpha|^{2} /|\alpha|^{3}
$$

whenever $\alpha, \beta \in \mathcal{V}_{m+1, x}$ and $\alpha \neq 0$. Note that the area integrand satisfies this condition with $c_{A}=1$.

LEMma. Let $T \in \mathbf{I}_{m+1}\left(M_{0}\right)$ be homologically $\Psi$-minimizing with respect to $M_{0}$, and suppose $g_{\sharp} T=T$ for $g \in G$. Then spt $T \sim \operatorname{spt} \partial T$ is an $(m+1)$-dimensional submanifold of class 2 of $M_{0}$. 
Proof. Assume spt $T \sim$ spt $\partial T \neq \varnothing$. Let $U$ be an open subset of the open set $M_{0}^{*} \sim \pi($ spt $\partial T)$ such that there exist an equivariant coordinate map $\phi_{U}: U \times$ $G / H \rightarrow \pi^{-1} U$ and a coordinate map $\chi: U \rightarrow \mathbf{R}^{n+1-m}$ with $\chi(U)=\mathbf{R}^{n+1-m}$. Inasmuch as $T$ is rectifiable and invariant, we infer using [FF, 8.16] that $T \wedge \xi=0$ for $\xi \in X_{G}(M)$, hence Corollary 4.2 implies that if $\mathbf{v}$ is a smooth unit $m$-vector field on $\pi^{-1} U$ which is tangent to the orbits, then $v$ is invariant. Using $v$ to define the integrand $\Psi^{*}$ of degree 1 on $U$ as in 2.5 we see that if $A^{*}$ is a compact subset of $U$ and $z \in A^{*}$ then

$$
D^{2} \Psi_{z}^{*}\left(\alpha^{*}\right)\left(\beta^{*}, \beta^{*}\right)>c_{\pi^{-1} A^{*}}\left|\beta^{*} \wedge \alpha^{*}\right|^{2} /\left|\alpha^{*}\right|^{3}
$$

for $\alpha^{*}, \beta^{*} \in \mathbf{T}_{z}\left(M_{0}^{*}\right), \alpha^{*} \neq 0$. In view of [F1, 5.1.3 and 5.1.2] we can therefore apply [F1, 5.3.20] in $U$ to conclude that if $R_{0} \in I_{1}(U)$ is absolutely $\Psi^{*}$-minimizing with respect to $U$, then spt $R_{0} \sim$ spt $\partial R_{0}$ is a 1-dimensional submanifold of class 2 of $M_{0}^{*}$.

Next apply Lemma 4.1 to obtain $R \in \mathscr{R}_{1}^{\text {loc }}(U)$ such that

$$
T \mid \mathscr{Q}^{m+1}\left(\pi^{-1} U\right)=\phi_{U \sharp}(R \times G / H),
$$

use [F1, 4.2.1, 4.3.4 and 4.3.6] to choose $r>0$ such that

$$
R_{r}=R\left\llcorner\chi^{-1}\{y:|y|<r\} \in \mathbf{I}_{1}(U),\right.
$$

and denote

$$
T_{r}=\phi_{U \sharp}\left(R_{r} \times G / H\right)=T L(\chi \circ \pi)^{-1}\{y:|y|<r\} .
$$

Referring to [F1, 4.1.28] we see that for $\|T\|$ almost all $x \in U$,

$$
\vec{T}(x)=\lambda_{x}(\vec{R}(\pi x)) \wedge \mathbf{v}(x),
$$

where $\lambda_{x}$ is chosen as in 2.5. Applying the coarea formula [F1, 3.2.22] we conclude that

$$
\pi_{\sharp}\left\|T_{r}\right\|=\left\|R_{r}\right\| L V
$$

and use this to verify that $\int_{T_{r}} \Psi=\int_{R_{r}} \Psi^{*}$. Finally, consider $Q \in \mathscr{Z}_{1}(U)=\mathscr{B}_{1}(U)$. Then

$$
S=\phi_{U \sharp}(Q \times G / H) \in \mathscr{B}_{m+1}\left(\pi^{-1} U\right),
$$

and reasoning as above with $T_{r}$ replaced by $T_{r}+S$ we obtain

$$
\int_{T_{r}+S} \Psi=\int_{R_{r}+Q} \Psi^{*}
$$

Consequently, $R_{r}$ is absolutely $\Psi^{*}$-minimizing with respect to $U$ and the remarks in the first paragraph imply that spt $R \cap \chi^{-1}\{y:|y|<r\}$ is smooth. We conclude that spt $R$, hence (spt $T) \cap\left(\pi^{-1} U\right)$, is smooth.

5.2. REMARK. The conclusion of the lemma also holds in case $m=n-2$ and $T \in \mathbf{I}_{n}\left(M_{0}\right)$. To verify this one uses [SSA, II.7] in place of [F1, 5.3.20] in the proof of the lemma. 
5.3. Corollary to Theorem 4.4. Assume $m \in\{n-1, n-2\}$ and $\Psi$ is elliptic and of class 3 on $M$. Then

$$
\text { spt } T \cap \text { int } K \cap M_{0} \sim \operatorname{spt} \partial T
$$

is an $n$-dimensional $G$-invariant submanifold of class 2 of $M$.

Proof. Using local coordinates in $W=\pi\left(K_{0} \sim\right.$ spt $\left.\partial T\right), K_{0}=$ int $K \cap M_{0}$, and using [F1, 4.2.1, 4.3.4 and 4.3.6] as in the proof of Lemma 5.1 we cover $W$ with open sets $U$ such that

$$
T_{U}=T\left\llcorner\pi^{-1} U \in \mathbf{I}_{n}(M), \quad \text { spt } T_{U} \subset K_{0}, \quad \text { spt } \partial T_{U} \subset \text { boundary } U .\right.
$$

Since $T_{U}$ is homologically $\Psi$-minimizing with respect to $K_{0}$ and is invariant by Theorem 4.4, we can apply Lemma 5.1 or Remark 5.2 with $M_{0}$ replaced by $K_{0}$ to infer that spt $T \cap U$ is smooth.

5.4. REMARK. For integrands which are close to the area integrand one has much more information concerning the regularity of spt $T$. Recall that $x \in \operatorname{spt} T \sim$ spt $\partial T$ is called singular if there is no neighborhood $U$ of $x$ such that $U \cap \operatorname{spt} T$ is a connected $n$-dimensional manifold of class 2 .

It is a consequence of [SSA, II.8] that there exists a neighborhood $\mathscr{U}$ of the area integrand in the class 2 topology such that the following is true: If $\Psi \in \mathscr{U}$ is a positive elliptic integrand of degree $n$ and class 3 in $M$ and $T \in \Re_{n}(M)$ is $G$-invariant and homologically $\Psi$-minimizing with respect to $M$, then no singular point of $T$ lies on an orbit of dimension greater than $n-7$.

6. Minimizing currents in $M_{0}^{*}$. Assume that $M_{0}^{*}$ is orientable (which follows in particular if $\mathbf{H}_{1}\left(M ; \mathbf{Z}_{2}\right)=0$ [BG, IV, 4.4]); this, together with orientability of $M_{0}$, implies that the standard fibre $Y=G / H$ and the bundle $\Re_{0}$ are orientable. Also assume that the action of $G$ on $Y$ preserves orientation; in view of Corollary 4.2 this assumption will result in no essential loss of generality, while the first example on [BG, p. 189] shows that it does not imply orientability of $M_{0}^{*}$. Denote $K^{*}=$ $\pi\left(K \cap M_{0}\right)$ and $L^{*}=\pi\left(L \cap M_{0}\right)$. Assume $\mathscr{H}^{n+1}(L)=0$.

6.1. Orientability of $\Re_{0}$ implies that the structure group $\Re(H) / H$ can be reduced to the subgroup $\mathcal{H}$ of orientation preserving transformations of $Y$. From now on we will regard $\mathcal{H}$ as the structure group of $\mathfrak{K}_{0}$; as remarked in 2.2, coordinate functions for $\mathfrak{T}_{0}$, hence admissible maps of the fibre $Y$, are equivariant.

Assign an $\mathcal{H}$-invariant metric to $Y$ such that $\mathcal{H}^{m}(Y)=1$, choose a unit orientation $\omega_{0} \in \mathscr{Q}^{m}(Y)$, and denote by $\vec{Y}$ the $m$-vector field on $Y$ such that $\left\langle\vec{Y}, \omega_{0}\right\rangle=1$. There exists a unique smooth unit $m$-vector field $\mathrm{v}$ on $M_{0}$ such that

$$
f_{\sharp} \vec{Y}=(V \circ \pi) \mathbf{v} \mid f(Y)
$$

whenever $f: Y \rightarrow M_{0}$ is admissible. Furthermore, $\mathbf{v}$ is $G$-invariant. Let $\Omega \in \mathcal{E}^{m}\left(M_{0}\right)$ be dual to $(V \circ \pi)$ v with respect to the metric $b$. Then $\Omega$ is $G$-invariant, $|\Omega|=$ $1 / V \circ \pi$, and $f^{\sharp} \Omega=\omega_{0}$ whenever $f$ is admissible. One uses $\Omega$ to show that the action of $G$ preserves orientation of $M$. 
The projection operator for $\mathfrak{T}_{0}$ is the continuous linear map

$$
P_{\mathscr{R}_{0}}: \mathscr{D}_{*}\left(M_{0}\right) \rightarrow \mathscr{D}_{*}\left(M_{0}^{*}\right)
$$

of degree $-m$ such that for $T \in \mathscr{D}_{k}\left(M_{0}\right)$,

$$
\begin{aligned}
& P_{\mathscr{N}_{0}}(T)=(-1)^{m(k-m)} \pi_{\sharp}(T L \Omega), \quad m<k, \\
& P_{\mathscr{T}_{0}}(T)=0, \quad m>k .
\end{aligned}
$$

The lifting operator for $\mathfrak{T K}_{0}$ is the linear map

$$
L_{\mathscr{T} R_{0}}: \mathbf{M}_{*}^{\mathrm{loc}}\left(M_{0}^{*}\right) \rightarrow \mathbf{M}_{*}^{\mathrm{loc}}\left(M_{0}\right)
$$

of degree $m$ characterized in [BD, 3.2]. $L_{\mathscr{T}_{0}}(R)$ is locally equal to the image of $R \times Y$ under a bundle coordinate map. Finally,

$$
P_{\mathscr{T}_{0}} \circ L_{\mathscr{T}_{0}}(R)=R
$$

whenever $R \in \mathbf{M}_{*}^{\text {loc }}\left(M_{0}^{*}\right)$. We remark that although $L_{\mathscr{T}_{0}}$ has a unique extension to a chain map defined on the chain complex spanned by $\mathbf{M}_{*}^{\text {loc }}\left(M_{0}\right)$ [BD, 3.3], such an extension does not exist for $P_{\mathscr{T}_{0}}$ unless $d \Omega=0$, which is true only if $\mathbf{H}^{m}\left(M_{0} ; \mathbf{R}\right) \neq$ 0 .

6.2. LemMa. Suppose $R_{0} \in M_{k}^{\mathrm{loc}}\left(M_{0}^{*}\right)$ and $T_{0}=L_{\mathscr{T}_{0}} R_{0}$. Then

(i) $T_{0} \in \mathbf{M}_{k+m}^{\text {loc }}\left(M_{0}\right)$ and $\pi_{\sharp}\left\|T_{0}\right\|=\left\|R_{0}\right\| \mathrm{L} V$,

(ii) for $\|T\|$ almost all $x \in M_{0}$,

$$
\vec{T}_{0}(x)=\bigwedge_{k} \lambda_{x}\left(\vec{R}_{0}(\pi x)\right) \wedge \mathrm{v}(x)
$$

whenever $\lambda_{x}$ is a linear right inverse of $\pi_{\#} \mid \mathbf{T}_{x}\left(M_{0}\right)$, and

(iii) $\int_{T_{0}} \Psi=\int_{R_{0}} \Psi^{*}$ whenever $\Psi$ is a positive integrand of degree $k+m$ on $M$.

Proof. (i) is [BD, 3.2 and 4.9] (for the proof of which it is not necessary to assume $G$ connected). (ii) follows from [BD, 3.3(4)]. (iii) follows from (i), (ii) and the definition of $\Psi^{*}$ in 2.5 .

6.3. LEMMA. Let $\Psi$ be a positive integrand of degree $k$ on $M$.

(i) To each $T_{0} \in \mathcal{R}_{k}^{\text {loc }}\left(M_{0}\right)$ with $\int_{T_{0}} \Psi<\infty$ and spt $T_{0} \subset K$ there corresponds a unique $T \in \mathscr{R}_{k}(M)$ such that

$$
T \mid \mathscr{D}^{k}\left(M_{0}\right)=T_{0} \text { and } \int_{T} \Psi=\int_{T_{0}} \Psi .
$$

(ii) Let $k=n$. Then $L_{\mathscr{T}_{0}}$ induces a one-to-one correspondence, also denoted $L_{\mathscr{T}_{0}}$, between

and

$$
\Re^{*}=\mathcal{R}_{n-m}^{\text {loc }}\left(M_{0}^{*}\right) \cap\left\{R: \int_{R} \Psi^{*}<\infty, \text { spt } R \subset K^{*}\right\}
$$

$$
\Re=\Re_{n}(M) \cap\left\{T: \text { spt } T \subset K, g_{\sharp} T=T \text { for } g \in G\right\} .
$$

(iii) Let $k=n$ and denote

$\mathscr{B}^{*}=\left\{Q+\partial P: P \in \mathscr{R}_{n-m+1}^{\text {loc }}\left(M_{0}^{*}\right),\|P\|(V)<\infty, Q \in \mathscr{F}_{n-m}^{\text {foc }}\left(M_{0}^{*}\right)\right.$, spt $\left.Q \subset L^{*}\right\}$.

Then

$$
L_{\mathscr{R}_{0}}\left(\mathscr{B}^{*} \cap \mathscr{R}^{*}\right)=\mathscr{B}_{n}(M, L) \cap \Re .
$$


Proof. Choose a neighborhood $U$ of $K$ and $\lambda>0$ so that $\|\alpha\|<\lambda \Psi(\alpha)$ for $x \in U$ and $\alpha \in \wedge_{k} \mathbf{T}_{x}(M)$. Then $\mathbf{M}\left(T_{0}\right) \leqslant \lambda \int_{T_{0}} \Psi<\infty$ and so there is a unique $T \in \mathbf{M}_{k}(M)$ such that

$$
T \mid \mathscr{D}^{k}\left(M_{0}\right)=T_{0} \quad \text { and } \quad\|T\|\left(M \sim M_{0}\right)=0 ;
$$

$T$ is clearly rectifiable. Moreover,

$$
\int_{T} \Psi-\int_{T_{0}} \Psi=\int_{M \sim M_{0}} \Psi(\vec{T}) d\|T\|,
$$

whence follows (i).

In case $k=n$ we infer using Lemma 3.5 that if $T_{0}$ is also invariant then $T_{0}$ is the restriction to $\mathscr{Q}^{n}\left(M_{0}\right)$ of a unique $T \in \Re_{n}(M) ; T$ is clearly invariant. Furthermore, it follows from [FF, 8.16], [BD, 4.2], Lemma 6.2(iii) and the proof of the last assertion of Lemma 4.1 that $L_{\mathscr{T}_{0}}$ maps $\Re^{*}$ univalently onto

$$
\mathcal{R}_{n}^{\mathrm{loc}}\left(M_{0}\right) \cap\left\{T_{0}: \int_{T_{0}} \Psi<\infty, g_{\sharp} T_{0}=T_{0} \text { for } g \in G, \text { spt } T_{0} \subset K\right\} \text {. }
$$

(ii) is now clear.

In order to verify (iii) we first consider $P, Q$ as in the definition of $\mathscr{B}^{*}$ with $Q+\partial P \in R^{*}$. Denoting $T=L_{\mathscr{T}_{0}}(Q+\partial P) \in \Re$ we infer using Lemma 6.2(i) that there exists $S \in \mathscr{R}_{n+1}(M)$ such that $S \mid \mathscr{Q}^{n+1}\left(M_{0}\right)=L_{\mathscr{T}_{0}} P$, hence

$$
\operatorname{spt}(\partial S-T) \subset L \cup\left(M \sim M_{0}\right) \text {. }
$$

Moreover, $\|S\|\left(M \sim M_{0}\right)=0$ because $\mathcal{F}^{n+1}\left(M \sim M_{0}\right)=0$, hence [BD, 4.2] implies that $S$ is invariant. Consequently, Lemma 3.5 implies that $\operatorname{spt}(\partial S-T) \subset L$.

On the other hand, suppose $\partial S+T \in \Re$ with $S \in \Re_{n+1}(M), T \in \mathscr{F}_{n}(M)$, spt $T \subset L$. Using Lemma 3.1 and the fact that $\mathcal{F}^{n+1}\left(L \cup\left(M \sim M_{0}\right)\right)=0$ we infer that $S$ is invariant hence there exists $P \in \Re_{n-m+1}^{\text {loc }}\left(M_{0}^{*}\right)$ such that $L_{\mathscr{T}_{0}} P=S \mid \mathscr{Q}^{n+1}\left(M_{0}\right)$; moreover, $\|P\|(V)<\infty$ by Lemma 6.2(i). Denoting $R=$ $P_{\mathscr{R}_{0}}(\partial S+T) \in R^{*}$ we conclude with the help of [BD, 3.3(8)] that

$$
T \mid \mathscr{Q}^{n}\left(M_{0}\right)=L_{\mathscr{T}_{0}}(R-\partial P)
$$

hence $\operatorname{spt}(R-\partial P) \subset L^{*}$.

6.4. TheOREM. Let $\Psi$ be a convex positive even $G$-invariant integrand of degree $n$ on M. Fix $0 \neq R \in R^{*}$ (in the notation of Lemma 6.3) and denote

$$
\mu^{*}=\inf \left\{\int_{R+Q} \Psi^{*}: Q \in \mathfrak{B}^{*} \cap \Re^{*}\right\}, \quad T=L_{\mathscr{T}_{0}} R \in \Re_{n}(M) .
$$

Assume $\mathcal{H}^{n}(B)=0$ where

$$
B=M_{0} \cap \operatorname{spt} \partial T \sim L=\pi^{-1}\left(\operatorname{spt} \partial R \sim L^{*}\right) .
$$

Then $T$ is homologically $\Psi$-minimizing in $K$ with respect to $(M, L)$ if and only if $\int_{R} \Psi^{*}=\mu^{*}$.

Proof. Suppose $\int_{R} \Psi^{*}=\mu^{*}$ and $T_{1} \in \Re_{n}(M)$ is homologically $\Psi$-minimizing in $K$ with respect to $(M, L)$ with $T-T_{1} \in \mathscr{B}_{n}(M, L)$ (see 2.4). Then

$$
M_{0} \cap \operatorname{spt} \partial T_{1} \sim L \subset B,
$$


and in view of the fact that $\|T\|\left(\left(M \sim M_{0}\right) \cup B\right)=0=\left\|T_{1}\right\|(B)$ by Lemma 6.3(i) and [F1, 4.1.20 and 2.10.6] we infer from [BD, 4.2] that $T$ satisfies the assumptions on $T^{\prime}$ in Lemma 4.3 with $T$ replaced by $T_{1}$. Consequently, Theorem 4.4 implies that $T_{1}$ is invariant, and referring to 6.1 and Lemma 6.3 we conclude that

$$
R_{1}=P_{\mathscr{R}_{0}} T_{1} \in \mathscr{R}_{n-m}^{\text {loc }}\left(M_{0}^{*}\right), \quad \text { spt } R_{1} \subset K^{*}, \quad R-R_{1} \in \Re^{*} \cap \Re^{*} .
$$

Thus by Lemma 6.2(iii),

$$
\int_{R_{1}} \Psi^{*} \geqslant \int_{R} \Psi^{*}=\int_{T} \Psi \geqslant \int_{T_{1}} \Psi=\int_{R_{1}} \Psi^{*}
$$

Finally, we suppose $T$ minimizes $\Psi$ and use Lemma 6.3 to infer that whenever $Q \in \mathscr{B}^{*} \cap \Re^{*}$,

$$
\int_{R} \Psi^{*}=\int_{T} \Psi \leqslant \int_{T+L_{9 \pi_{0}} Q} \Psi=\int_{R+Q} \Psi^{*}
$$

6.5. REMARK. It follows from [F1, 2.10.25 and 2.10.45] that

$$
\mathcal{H}^{n}(B)=0 \quad \text { if and only if } \mathcal{H}^{n-m}(\pi B)=0 .
$$

\section{Examples.}

7.1. An orthogonal action of $\mathbf{S}^{1}$ on $\mathbf{R}^{3}$ can be represented as the action of a one-parameter subgroup $G$ of $\mathbf{S O}(3)$. The members of $G$ have a common eigenvector $v$; denote by $\Lambda$ the linear subspace spanned by $v$. Then $G$ is the group of rotations of $\mathbf{R}^{3}$ about $\Lambda$. Let $c_{1}$ and $c_{2}$ be circles centered on $\Lambda$ lying in planes orthogonal to $\Lambda$ and oriented in the same way. Let $k_{1}$ and $k_{2}$ be integers, $Q=k_{1} c_{1}+k_{2} c_{2}$, and $T$ be an absolutely area minimizing rectifiable current with $\partial T=Q$. Then by $[\mathbf{F 1}, 5.4 .15]$, spt $T \sim\left(c_{1} \cup c_{2}\right)$ is a smooth 2-dimensional minimal submanifold of $\mathbf{R}^{3}$ which is a surface of revolution by Theorem 4.4. It is an elementary exercise in differential geometry to show that spt $T$ is a subset of the union of a catenoid and the discs spanned by $c_{1}$ and $c_{2}$.

7.2. Let $\mathbf{S}^{3}=\mathbf{C}^{2} \cap\left\{(z, w):|z|^{2}+|w|^{2}=1\right\}$ and $k_{2}>k_{1}>0$ be relatively prime integers. We define an orthogonal action of $\mathbf{S}^{\mathbf{1}}$ on $\mathbf{S}^{3}$ by means of the function which for $\alpha \in \mathbf{R}$ maps $(z, w)$ to $\left(e^{i k_{1} \alpha} z, e^{i k_{2} \alpha} w\right)$, and denote the corresponding subgroup of $\mathbf{S O}(4)$ by $G_{k_{1}, k_{2}}$. Each $G_{k_{1}, k_{2}}$ represents a distinct action of $\mathbf{S}^{1}$ on $\mathbf{S}^{3}$, and each closed one-parameter subgroup of $\mathbf{S O}(4)$ is conjugate to some $G_{k_{1}, k_{2}}$.

Let $c_{1}$ and $c_{2}$ be oriented geodesic circles in $\mathbf{S}^{3}$ which are invariant under the action of $G_{k_{1}, k_{2}}, p$ and $q$ be integers, $Q=p c_{1}+q c_{2}$, and $T$ be an absolutely area minimizing rectifiable current with $\partial T=Q$. In contrast with the preceding example the existence of inequivalent actions of $\mathbf{S}^{1}$ on $\mathbf{S}^{3}$ gives rise to many more possibilities for $T$; in fact, there may exist no $G_{k_{1}, k_{2}}$-invariant $T$. The case where

$$
c_{1}=\mathbf{S}^{3} \cap \mathbf{C} \times\{0\}, \quad c_{2}=\mathbf{S}^{3} \cap\{0\} \times \mathbf{C}
$$

is particularly interesting because in this case $Q$ is $G_{k_{1}, k_{2}}$-invariant for all choices of $k_{1}, k_{2}$. We assume the orbits of $G_{k_{1}, k_{2}}$ to have the orientation induced by the natural orientation of $\mathbf{S}^{1}$; this means in particular that $c_{1}$ and $c_{2}$ have their usual orientations when regarded as subsets of $\mathbf{C}$. 
As we saw in $\S 6$, in order to understand the structure of an invariant $T$ it is sufficient to study the solutions to a suitable one-dimensional variational problem in the orbit space $X_{k_{1}, k_{2}}=\mathbf{S}^{3} / G_{k_{1}, k_{2}}=\pi_{k_{1}, k_{2}}\left(\mathbf{S}^{3}\right)$. The structures of these spaces are presented in detail in [HL, Chapters III and IV]; assuming $k_{1}>k_{2}>0$ we summarize the discussion therein:

(1) $X_{k_{1}, k_{2}}$ is a smooth manifold which is diffeomorphic to $\mathbf{S}^{2}$.

(2) For $k_{1}=k_{2}=1$ all orbits are principal and the projection $\pi_{1,1}: \mathbf{S}^{3} \rightarrow X_{1,1}$ is the Hopf map.

(3) For $k_{1}>k_{2}>1$ all orbits except $c_{1}$ and $c_{2}$ are principal and have trivial isotropy groups. $c_{1}$ and $c_{2}$ are exceptional orbits with isotropy groups $\mathbf{Z}_{k_{1}}$ and $\mathbf{Z}_{k_{2}}$, respectively. If $k_{2}=1$ then $c_{2}$ is a principal orbit.

(4) Define $\psi: \mathbf{R} \times(0, \pi) \rightarrow \mathbf{S}^{3}$ by

$$
\psi(\theta, \phi)=\left(\cos (\phi / 2), e^{i \theta} \sin (\phi / 2)\right) .
$$

Then $\pi_{k_{1}, k_{2}} \circ \psi$ is an immersion onto $X_{k_{1}, k_{2}} \sim\left\{c_{1}, c_{2}\right\}$.

(5) With respect to the coordinates induced by $\pi_{k_{1}, k_{2}} \circ \psi$ the metric $b_{\pi}$ of 2.2 is given by the formula

$$
\frac{1}{4}\left[k_{1}^{2} \sin ^{2} \phi\left(k_{1}^{2} \cos ^{2}(\phi / 2)+k_{2}^{2} \sin ^{2}(\phi / 2)\right)^{-1} d \theta^{2}+d \phi^{2}\right] .
$$

Further, the volume function is

$$
V(\theta, \phi)=2 \pi\left(k_{1}^{2} \cos ^{2}(\phi / 2)+k_{2}^{2} \sin ^{2}(\phi / 2)\right)^{1 / 2} .
$$

(6) If $\Psi$ is the two-dimensional area integrand on $\mathbf{S}^{3}$, then the associated integrand $\Psi^{*}$ on $X_{k_{1}, k_{2}} \sim\left\{c_{1}, c_{2}\right\}$ is $\sqrt{\pi / 2}$ times the length integrand with respect to the metric

$$
\begin{aligned}
b^{*}(\theta, \phi) & =(2 / \pi)\left(V^{2} b_{\pi}\right)(\theta, \phi) \\
& =k_{1}^{2} \sin ^{2} \phi d \theta^{2}+\left(k_{1}^{2} \cos ^{2}(\phi / 2)+k_{2}^{2} \sin ^{2}(\phi / 2)\right) d \phi^{2} .
\end{aligned}
$$

(7) $b^{*}$ extends to a metric of class 2 on $X_{k_{1}, k_{2}}$ which is the metric of a surface of revolution on which $c_{1}$ and $c_{2}$ correspond to axis points and $\phi$ and $\theta$ respectively parametrize the longitudes and meridians. In case $k_{1}=1=k_{2}, b^{*}$ is the metric of the unit sphere $\mathbf{S}^{2}$ in $\mathbf{R}^{3}$.

(8) The normalizer $\Re\left(G_{k_{1}, k_{2}}\right)$ of $G_{k_{1}, k_{2}}$ in $\mathbf{S O}(4)$ is the torus acting on $\mathbf{S}^{3}$ by means of the function which for $(u, v) \in \mathbf{R}^{2}$ maps $(z, w)$ to $\left(e^{i u} z, e^{i v} w\right)$. The group $\Re\left(G_{k_{1}, k_{2}}\right) / G_{k_{1}, k_{2}}$ acts on $X_{k_{1}, k_{2}}$ and preserves the coordinate $\phi$, hence acts as the group of revolutions of $X_{k_{1}, k_{2}}$ (about the axis through $c_{1}$ and $c_{2}$ ).

(9) For each $a \in \mathbf{R}$ the longitude $\gamma_{a}$ consisting of the closure of these points of $X_{k_{1}, k_{2}} \sim\left\{c_{1}, c_{2}\right\}$ with $\theta$ coordinate equal to $a$ is (when properly parametrized) a geodesic on $X_{k_{1}, k_{2}}$ with endpoints $c_{1}, c_{2} \cdot \gamma_{a} \cup \gamma_{\pi+a}$ is a closed geodesic whose inverse image $\tau_{k_{1}, k_{2} ; a}=\pi_{k_{1}, k_{2}}^{-1}\left(\gamma_{a} \cup \gamma_{\pi+a}\right)$ is a minimally immersed torus, and any two of these surfaces are congruent. In particular, $\tau_{k_{1}, k_{2} ; 0}$ is the real algebraic variety

$$
\mathbf{S}^{3} \cap\left\{(z, w): \operatorname{Im} z^{k_{2}} \bar{w}^{k_{1}}=0\right\}
$$


discussed in [L1, §7]. $\tau_{1,1 ; a}$ is congruent to the Clifford torus

$$
\mathbf{S}^{3} \cap\{(z, w):|z|=|w|\} .
$$

The immersion $\Phi: \mathbf{R}^{2} \rightarrow \mathbf{S}^{3}$ defined by

$$
\Phi(\phi, \alpha)=\left(e^{i k_{1} \alpha} \cos (\phi / 2), e^{i\left(k_{2} \alpha+a\right)} \sin (\phi / 2)\right)
$$

maps $\mathbf{R}^{2}$ onto $\tau_{k_{1}, k_{2} ; a}$. Moreover,

$$
\sigma_{k_{1}, k_{2} ; a}=\pi_{k_{1}, k_{2}}^{-1}\left(\gamma_{a}\right) \sim\left(c_{1} \cup c_{2}\right)
$$

is the image under $\Phi$ of $(0, \pi) \times[0,2 \pi)$, hence is an embedded cylinder. We orient $\sigma_{k_{1}, k_{2} ; a}$ so that the current

$$
\sigma_{k_{1}, k_{2} ; a}=\Phi_{\sharp}((0, \pi) \times[0,2 \pi)) ;
$$

this implies that

$$
\partial \sigma_{k_{1}, k_{2} ; a}=k_{2} c_{2}-k_{1} c_{1} .
$$

Finally, note that $\sigma_{k_{1}, k_{2} ; a}$ is $G_{k_{1}, k_{2}}$-invariant and that if $a_{1}, a_{2} \in \mathbf{R}$ then there exists $F \in \mathbf{S O}(4)$ such that $F_{\sharp} \sigma_{k_{1}, k_{2} ; a_{1}}=\sigma_{k_{1}, k_{2} ; a_{2}}$.

THEOREM. Let $T \in \mathscr{R}_{2}\left(\mathbf{S}^{3}\right)$ be absolutely area minimizing with spt $\partial T=c_{1} \cup c_{2}$, and $k_{1}, k_{2}$ be relatively prime positive integers. Then $T$ is $G_{k_{1}, k_{2}}$-invariant if and only if there exists an integer $r$ such that

$$
\partial T=r\left(k_{2} c_{2}-k_{1} c_{1}\right)
$$

If $T$ is $G_{k_{1}, k_{2}}$-invariant, then there exist

$$
\beta= \pm 1, \quad a_{j} \in \mathbf{R}, \quad 0<\alpha_{j} \in \mathbf{Z}, \quad j=1, \ldots, \nu,
$$

such that $r=\beta\left(\alpha_{1}+\cdots+\alpha_{v}\right)$ and

$$
T=\beta \sum_{j=1}^{\nu} \alpha_{j} \sigma_{k_{1}, k_{2} ; a_{j}}
$$

Proof. Assuming $\partial T=r\left(k_{2} c_{2}-k_{1} c_{1}\right)$ we apply Theorem 4.4 with $T^{\prime}=r \sigma_{k_{1}, k_{2} ; a}$ and conclude that $T$ is invariant.

In that which follows we always refer to the metric $b^{*}$ on $X_{k_{1}, k_{2}}$. Setting $K=\mathbf{S}^{3}$ we denote the lifting operator of Lemma 6.3(ii) by $L_{k_{1}, k_{2}}$, or simply $L$, where the form $\omega_{0} \in \mathscr{Q}^{1}\left(\mathbf{S}^{1}\right)$ used in defining $L$ (see 6.1 ) is positively oriented with respect to the usual orientation of $\mathbf{S}^{1}$. Observing that

$$
\Re^{*}=\left\{R \mid \mathscr{D}^{1}\left(X_{k_{1}, k_{2}} \sim\left\{c_{1}, c_{2}\right\}\right): R \in \mathscr{R}_{1}\left(X_{k_{1}, k_{2}}\right)\right\}
$$

we see that $L_{k_{1}, k_{2}}$ induces an isomorphism

$$
L_{k_{1}, k_{2}}: \Re_{1}\left(X_{k_{1}, k_{2}}\right) \rightarrow \Re_{2}\left(\mathbf{S}^{3}\right) \cap\left\{T^{\prime}: T^{\prime} \text { is } G_{k_{1}, k_{2}} \text {-invariant }\right\} .
$$

Furthermore, in this case the set $\mathscr{B}^{*}$ of Lemma 6.3(iii) (with $L=\varnothing$ ) is equal to $\mathscr{B}_{1}\left(X_{k_{1}, k_{2}}\right)$. Finally, we observe that if $\gamma_{a}$ is oriented so that $\partial \gamma_{a}=c_{2}-c_{1}$ then the current $\sigma_{k_{1}, k_{2} ; a}=L_{k_{1}, k_{2}}\left(\gamma_{a}\right)$. (See [BJ1, 3.5(6)].)

Suppose $T$ is $G_{k_{1}, k_{2}}$-invariant. Then there exists $R \in \Re_{1}\left(X_{k_{1}, k_{2}}\right)$ such that $T=$ $L(R)$; moreover, $R$ minimizes length by Theorem 6.4. [F1, 5.3.20] implies that spt $R \sim\left\{c_{1}, c_{2}\right\}$ is smooth, hence there exist

$$
a_{j} \in \mathbf{R} \text { and } 0 \neq \beta_{j} \in \mathbf{Z}, \quad j=1, \ldots, \nu,
$$


such that

$$
R=\beta_{1} \gamma_{a_{1}}+\cdots+\beta_{\nu} \gamma_{a_{i}}
$$

Thus

$$
T=\sum_{j=1}^{\nu} \beta_{j} \sigma_{k_{1}, k_{2} ; a_{j}}
$$

and

$$
\partial T=\sum_{j=1}^{\nu} \beta_{j}\left(k_{2} c_{2}-k_{1} c_{1}\right) ;
$$

inasmuch as $T$ minimizes area, the latter expression implies that all $\beta_{j}$ have the same sign.

REMARKs. (1) It follows in particular from the theorem that although $p c_{1}+q c_{2}$ $\in \Re_{1}\left(S^{3}\right)$ is invariant under the action of every $G_{k_{1}, k_{2}}$, for a given pair of nonzero integers $p, q$ there may exist no $T \in \mathscr{R}_{2}\left(\mathbf{S}^{3}\right)$ with $\partial T=p c_{1}+q c_{2}$ which is invariant under a given action.

(2) The classification of those area minimizing rectifiable currents $T$ with $\partial T=r c_{1}$ can be carried out in a manner similar to the above through application of our theory to the group $G_{r, 0}$ using the discussion of the orbit space $\mathbf{S}^{3} / G_{r, 0}$ in [HL, Chapter III]. However, the structure of such currents also follows directly from [BJ2, 4.5]; in particular, the group action is irrelevant:

There exist oriented two-dimensional great hemispheres $\sigma_{1}, \ldots, \sigma_{\nu}$ and

$$
\beta= \pm 1, \quad 0<\alpha_{j} \in \mathbf{Z}, \quad j=1, \ldots, \nu
$$

such that $\partial \sigma_{j}=c_{1}, r=\beta\left(\alpha_{1}+\cdots+\alpha_{\nu}\right)$ and

$$
T=\beta \sum_{j=1}^{\nu} \alpha_{j} \sigma_{j}
$$

(3) Finally, to complete the classification of those area minimizing rectifiable currents $T$ with $\partial T=p c_{1}+q c_{2}$, we observe that if $p q>0$, then

$$
\partial F_{\sharp} T=q c_{2}-p c_{1}
$$

where $F$ is the isometry of $C^{2}$ such that $F(z, w)=(\bar{z}, w)$. The structure of $F_{\sharp} T$ can now be inferred from the theorem.

7.3. Let $M=\mathbf{R}^{\lambda} \times \mathbf{R}^{\lambda}=\mathbf{R}^{2 \lambda}, \lambda \geqslant 2$, and $G$ be the subgroup of $\mathbf{O}(2 \lambda)$ generated by $\operatorname{SO}(\lambda) \times \operatorname{SO}(\lambda) \cup\{\alpha\}$, where we define

$$
\begin{aligned}
\sigma(x) & =\left(x^{1},-x^{2}, \ldots,-x^{\lambda}\right) \in \mathbf{R}^{\lambda} \text { for } x \in \mathbf{R}^{\lambda}, \\
\alpha(x, y) & =(y, \sigma(x)) \in \mathbf{R}^{\lambda} \times \mathbf{R}^{\lambda} \quad \text { for }(x, y) \in \mathbf{R}^{\lambda} \times \mathbf{R}^{\lambda} .
\end{aligned}
$$

We also denote by $\operatorname{SO}(\lambda-1)^{\prime}$ the isotropy subgroup at $e_{1}$ of the action of $\operatorname{SO}(\lambda)$ on $S^{\lambda-1}$. Note that each isotropy subgroup $G_{(x, y)}$ is conjugate to $G_{\left(|x| e_{1}|y| e_{1}\right)}$. In view of the fact that $\alpha$ is in the normalizer of $\operatorname{SO}(\lambda) \times \operatorname{SO}(\lambda)$ it is not difficult to verify that for $r, s \in R, G_{\left(r e_{1}, s e_{1}\right)}$ is generated by 


$$
\begin{array}{ll}
\mathbf{S O}(\lambda-1)^{\prime} \times \mathbf{S O}(\lambda-1)^{\prime} \cup\left\{\alpha^{2}\right\}, & \text { for } 0 \neq r \neq s \neq 0, \\
\mathbf{S O}(\lambda-1)^{\prime} \times \mathbf{S O}(\lambda-1)^{\prime} \cup\{\alpha\}, & \text { for } r=s \neq 0, \\
\mathbf{S O}(\lambda-1)^{\prime} \times \mathbf{S O}(\lambda) \cup\left\{\alpha^{2}\right\}, & \text { for } r \neq 0, s=0, \\
G, & \text { for } r=s=0 .
\end{array}
$$

Consequently, the orbit $G(x, y)$ is principal for $0 \neq|x| \neq|y| \neq 0$, special exceptional for $|x|=|y| \neq 0$, and singular for $|x||y|=0$ (but in verifying this one must keep in mind that $\alpha^{2} \in \mathbf{S O}(\lambda-1)^{\prime} \times \mathbf{S O}(\lambda-1)^{\prime}$ if and only if $\lambda$ is odd).

Inasmuch as $G$ is not connected, the principal orbits are not connected. Furthermore, the union of the set of nonprincipal orbits contains

$$
S=\mathbf{R}^{\lambda} \times \mathbf{R}^{\lambda} \cap\{(x, y):|x|=|y|\},
$$

which contains the support of a nonzero invariant rectifiable current of dimension $2 \lambda-1$, hence the conclusion of the fundamental Lemma 3.5 is not true. Note also that $G$ does not lie in $\mathbf{S O}(2 \lambda)$, hence the proposition in [L2] asserting existence of invariant solutions to the least area problem does not apply.

Assign an orientation to $\mathbf{S}^{\lambda-1}$, denote

$$
Q=\mathbf{S}^{\lambda-1} \times \mathbf{S}^{\lambda-1} \in \Re_{2 \lambda-2}(M),
$$

and suppose $T \in \mathscr{R}_{2 \lambda-1}(M)$ is absolutely area minimizing with $\partial T=Q$. It is shown in [F1, 5.4.17] that if $T$ were $G$-invariant, then spt $T$ would be equal to $S \cap\{(x, y):|x| \leqslant 1\}$, a contradiction to the regularity theorem [F1, 5.4.15] in case $\lambda=2$ or $\lambda=3$.

\section{REFERENCES}

[BD] D. Bindschadler, Invariant solutions to the oriented Plateau problem in a class of Riemannian manifolds, Trans. Amer. Math. Soc. (to appear).

[BG] G. Bredon, Introduction to compact transformation groups, Academic Press, New York, 1972.

[BJ1] J. E. Brothers, Integral geometry in homogeneous spaces, Trans. Amer. Math. Soc. 124 (1966), 480-517.

[BJ2] _ The structure of solutions to Plateau's problem in the n-sphere, J. Differential Geometry 11 (1976), 387-400.

[F1] H. Federer, Geometric measure theory, Springer-Verlag, Berlin and New York, 1969.

[F2] _. The singular sets of area minimizing rectifiable currents with codimension one and of area minimizing flat chains modulo two with arbitrary codimension, Bull. Amer. Math. Soc. 76 (1970), 767-771.

[F3] __ Real flat chains, cochains and variational problems, Indiana Univ. Math. J. 24 (1974), 351-407.

[FF] H. Federer and W. H. Fleming, Normal and integral currents, Ann. of Math. (2) 72 (1960), 458-520.

[HL] W. Y. Hsiang and H. B. Lawson, Jr., Minimal submanifolds of low cohomogeneity, J. Differential Geometry 5 (1971), 1-38.

[KN] S. Kobayashi and K. Nomizu, Foundations of differential geometry. I, Interscience, New York, 1963.

[L1] H. B. Lawson, Jr., Complete minimal surfaces in $\mathbf{S}^{3}$, Ann. of Math. (2) 92 (1970), 335-374.

[L2] _ The equivariant Plateau problem and interior regularity, Trans. Amer. Math. Soc. 173 (1972), 231-249.

[SSA] R. Schoen, L. Simon and F. J. Almgren, Jr., Regularity and singularity estimates on hypersurfaces minimizing parametric elliptic variational integrals, Acta Math 139 (1977), 217-265.

Department of Mathematics, Indiana University, Bloommgton, Indiana 47401 